"Agitation and End-of-Life: Towards an Advance Directive that Prepare for Agitation and Behavioral Symptoms in Alzheimer's Disease" proposes a framework for how Advance Directives can prepare for the unique decisions that arise as a person experiences agitation and other behavioral symptoms of Alzheimer's.

The framework proposed in this project draws from the recent development of Psychiatric Advance Directives led in part by the American Psychiatric Association, which have pioneered the use of Advance Directives for anticipated behavioral challenges. Specifically, Psychiatric Advance Directives allow individuals to specify in advance which treatments may be administered in response to acute episodes of psychiatric illness at a time when someone is unable or unwilling to provide consent. Our project contends that the mechanisms underlying Psychiatric Advance Directives be modeled but modified to help people, families, and providers prepare for agitation and the behavioral aspects of Alzheimer's.

Specifically, we propose a four-part framework for Advance Directives to prepare for agitation and other behavioral aspects of Alzheimer's:

1. Psychiatric medications. What treatments may - or may not - be used to manage agitation or other behavioral disturbances?

2. Agitation prevention and de-escalation. What strategies and techniques can caregivers employ to mollify agitated behaviors? How should caregivers respond to episodes of agitation?

3. Lifestyle preferences and values. What values - religious or otherwise - should guide care and treatment?

4. Information sharing and access. When and how should caregivers, medical professionals, and family members be notified - or share information about - behavioral disturbances?

It is well established in the scientific and medical literature that agitation and behavioral aspects of Alzheimer's can cause severe difficulty for families as the disease progresses. Advance Directives that prepare for agitation can help to create a plan and ease the challenges prompted by agitation and other behavioral aspects of Alzheimer's.

\title{
534 - Grief in de elderly and its impact
}

\section{Caldas ${ }^{1}$}

${ }^{1}$ Medical trainee at Centro Hospitalar Psiquiátrico de Lisboa, Lisbon, Portugal

Introduction With coronavirus disease 2019 (COVID-19) a lot of people have succumbed and older adults were disproportionately affected. $(1,2)$ Also, grief is encountered frequently in clinical practice with the elderly, and can be defined as the natural response to the death of a loved one. Still, when a failure of adaptation occurs then a Prolonged Grief Disorder (PGD) can be diagnosed, which has been recently included in ICD-11. The principal feature of PGD is a persistent preoccupation or an intense longing for the deceased that lasts longer than 6 months after the death. (3)

Objective: Comprehend the grief in the elderly and its implications in their health.

Method: The author conducted a literature review by searching the Pubmed database using the keywords elderly; grief; complicated grief; covid-19

Results: PGD is relatively prevalent among the elderly and has been associated with some variables related to the death time of the deceased, being the prevalence inversely correlated with the deceased's. $(4,5)$ Additionally, older adults present a higher risk of developing PGD when they are less 
educated, male, have poorer cognitive performance, and a history of depression. (3) PGD is characterized by sadness, fascination with or excessive avoidance of the memories associated with the deceased, the memory of the loss and mental ruminations about death. According to some investigations, PGD reduces life expectancy, increasing the odds of death, causes severe behavioural symptoms, and is associated with longer-term functional impairment. $(4,5)$

Conclusion: Given these results, it is easy to understand the need for a closer monitoring of these patients during this global crisis. Moreover, it is also important to find new ways to do that while complying with COVID-19 rules and restrictions. A short-term evidence-based intervention for PGD effective in the elderly is based on support focused upon these seven themes: understanding their grief, managing emotional pain, thinkingabout the future, strengthening their relationships, telling the story of the dead, learning to live with reminders of the deceased, and connecting with memories. (3) 1 Goveas, JS, Shear, MK. Grief and the COVID-19 Pandemic in Older Adults. Am J of Geriatric Psychiatry (2020). 28:10,1119-1125. https://doi.org/10.1016/j.jagp.2020.06.021

2 Ishikawa, RZ. I May Never See the Ocean Again: Loss and Grief Among Older Adults During the COVID-19 Pandemic.J. Psychol. Trauma (2020) Vol. 12, No. S1, S85-S86. http://dx.doi.org/10.1037/tra0000695

3 Meichsner, F, O'Connor, M. et al. Grief Before and After Bereavement in the Elderly: An Approach to Care. Am J ofGeriatric Psychiatry (2020). 28:5, 560-569. https://doi.org/10.1016/j.jagp.2019.12.010

4 Prigerson, HG., Frank, E. et al. Complicated Grief and Bereavement-Related Depression as Distinct Disorders:Preliminary Empirical Validation in Elderly Bereaved Spouses. AmJPsychiatry (1995) 152:1

5 Morowatisharifabad, MA., Alizadeh, A. et al. Prevalence of complicated grief and related factors in elderly individuals in Sabzevar City, Iran. Psycogeraitrics (2020). doi:10.1111/psyg.12579

\section{5 - Loneliness and social isolation in attendees of a Psychiatry of Old Age Service in the North West of Ireland: preliminary findings of a cross-sectional study during the COVID- 19 pandemic.}

\section{Author List}

Clodagh Rushe, Bernadette Kevin, Ericka Maye, Gavin Sweeney, Kevin McLaughlin, Marguerite Cryan, Aislinn Gannon, Vincent Melvin, Valerie Cogan, Donna Wilkie, Elaine McDonagh, Geraldine McCarthy, Catherine Dolan.

\section{Aims and Hypothesis}

The primary aim is to establish the prevalence of loneliness and social isolation in older adults referred to a Psychiatry of Old-Age Service in the North-West of Ireland. Secondary aims include exploring associations between loneliness, social isolation and, well-being, depression, cognition and healthstatus. The overarching aim was to improve patient outcomes by tailoring mental health and social interventions appropriate to patient need.

\section{Background}

Loneliness is a discrepancy between the social-relations one has and their desired level. It is estimated that one third of older adults will experience loneliness, which along with social isolation has links to poorer health-outcomes, reduced quality of life and cognitive decline. Government advice in Ireland to reduce social activity due to COVID-19 pandemic may compound social disconnection. We present preliminary findings of an ongoing study investigating loneliness, social isolation and related factors in older adults referred to a Mental-Health Service in the North-West of Ireland in 2020 - 2021. 